\title{
Fouling-Release Properties of Dendritic Polyglycerols against Marine Diatoms
}

Robin Wanka ${ }^{a}$, John A. Finlay ${ }^{b}$, Kim A. Nolte ${ }^{a}$, Julian Koc ${ }^{a}$, Victoria Jakobi ${ }^{a}$, Charlotte Anderson $^{b}$, Anthony S. Clare ${ }^{b}$, Harrison Gardner ${ }^{c}$, Kelli Z. Hunsucker ${ }^{c}$, Geoffrey W. Swain ${ }^{c}$, and Axel Rosenhahn ${ }^{a^{*}}$

a Analytical Chemistry - Biointerfaces, Ruhr-University Bochum, 44780 Bochum, Germany

b School of Natural and Environmental Sciences, Newcastle University, Newcastle upon Tyne NE1 7RU, United Kingdom

c Center for Corrosion and Biofouling Control, Florida Institute of Technology, Melbourne, FL, 32901, USA 


\section{Abstract}

Dendritic polyglycerols (PGs) were grafted onto surfaces using a ring-opening polymerization reaction and the fouling-release properties against marine organisms were determined. The coatings were characterized by spectroscopic ellipsometry, contact angle goniometry, ATR-FTIR and stability tests in different aqueous media. A high resistance towards the attachment of different proteins was found. The PG coatings with three different thicknesses were tested in a laboratory assay against the diatom Navicula incerta and in a field assay using a rotating disk. Under static conditions, the PG coatings did not inhibit the initial attachment of diatoms, but up to $94 \%$ of attached diatoms could be removed from the coatings after exposure to a shear stress of $19 \mathrm{~Pa}$. Fouling-release was found to be enhanced if the coatings were sufficiently thick. The excellent fouling-release properties were supported in dynamic field-immersion experiments in which the samples were continually exposed to a shear stress of $0.18 \mathrm{~Pa}$.

Keywords: antifouling, glycidol, hyperbranched, biofilms, biofouling, navicula incerta 


\section{Introduction}

Marine biofouling is the undesirable growth and adhesion of naval organisms on submerged surfaces and represents a ubiquitous problem with both environmental and economic impacts. ${ }^{1,2}$ Since the use of biocidal antifouling paints is being increasingly restricted, science and industry are seeking environmentally friendly coatings to combat fouling. A combination of surface properties is required to produce inert surfaces against biofouling, including optimized chemistry, charge, morphology, surface tension and elastic modulus. ${ }^{1,3,4}$ Polyethylene glycols (PEG) are a unique class of fouling-resistant materials that find application in sensors, ${ }^{5}$ implant technology ${ }^{6}$ and marine coatings. ${ }^{7,8}$ The high resistance of this material is frequently assigned to its inherent charge neutrality and the high hydration of the coatings. ${ }^{9}$ The PEG components have proven particularly effective in amphiphilic silicon coatings. ${ }^{10,11}$ Silicones and fluoropolymers are established ingredients of fouling-release (FR) coatings capable of weakening the adhesion between marine organisms and the surface. The motion of a ship moving through water causes the fouling organisms to be removed from the surface of the hull. Good FR properties are typically related to the surface energy, smoothness and thickness of the coating. ${ }^{12}$ Many modern FR systems are not only based on hydrophobicity, but add hydrophilic components, such as PEG, to achieve amphiphilic properties. ${ }^{13}$ In addition to linear block-copolymers, hyperbranching polymers (e.g. dendritic) have the advantage of possessing a high density of functional groups and tend to form a strong three-dimensional network. ${ }^{14}$ Hyperbranched fluoropolymers (HBFPs) show excellent protein resistance ${ }^{11,15,16}$ and inhibit the settlement of cypris larvae, ${ }^{17}$ although the prevention of diatom adhesion remains a challenge. ${ }^{10}$ Both block-copolymers and HBFP benefit from enhanced ethylene glycol content. ${ }^{10,18}$ Surfaces coated with PEG, particularly in long-term applications, tend 
to show oxidative and thermal degradation effects, especially when trace metals are present. ${ }^{19-21}$ UV-light is known to accelerate this degradation..$^{22,23}$

Dendritic polyglycerols (PGs) have been shown previously to resist protein adsorption. ${ }^{24}$ Their high biocompatibility was demonstrated in both in vivo and in vitro assays. ${ }^{25}$ Theoretical modeling of the structure of tethered Star-PEG copolymers using mean field theory analysis revealed that the polymers are able to form dense layers even at moderate grafting densities. The distribution within the film can be influenced by the distribution of the tether segments. ${ }^{26}$ Star-PEGs consist of a six-arm star-shaped polyether with an ethylene/propylene oxide backbone. Each star molecule possesses six reactive isocyanate end groups enabling modification and cross-linking. Star-PEGs offer a higher density of functional groups which are theoretically available for cross-linking. ${ }^{27-29}$ However, coated surfaces suffer frequently from an uneven segment density. This uneven density is considered to be a potential reason why linear PEGs achieved better results in protein resistance tests. ${ }^{30}$ Promising candidates for a more even segment distribution are dendritic PG coatings, as introduced by Haag et al. ${ }^{24}$ Nucleophiles can evoke a surface-initiated chain growth by a ringopening polymerization (ROP) of glycidol (2-hydroxymethyloxirane), depicted in Figure 1A. 


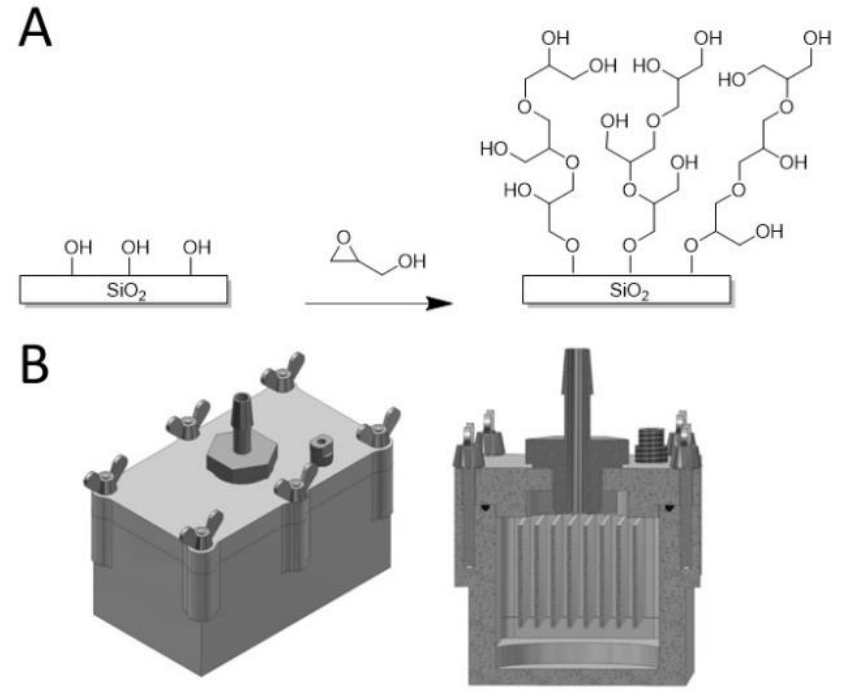

Figure 1. A: Summary of the ring-opening polymerization (ROP) of glycidol on a hydroxylated silica surface. The $\mathrm{OH}$ groups of the silicon oxide act as a nucleophile and can promote the grafting process of the dendritic polyglycerol (PG). B: Teflon reaction vessel developed for the ROP synthesis of PGs on glass slides under a

protective gas atmosphere. A fiberglass-enhanced Teflon was used to reinforce the mechanical stability during high temperature treatment. Six thumbscrews distributed the pressure equally to the bottom, to prevent deformation of the box. An internal temperature sensor allowed control of the reaction conditions. A free space is left at the bottom for a magnetic stirring bar which enables thorough mixing of glycidol and N- methyl-2-pyrrolidone. An argon-filled balloon was attached to the tap on the top during the reaction, which served as a pressure equalizer and inert gas reservoir.

Furthermore, dendritic PGs have the advantage of being more resistant to high temperature, oxidation and radiation. ${ }^{31}$ Polyglycerol has been used in recent years to generate dendrimer-like SAM systems on gold ${ }^{31,32}$ or as a triethoxysilane coupled compound to cover glass surfaces. ${ }^{33}$ Both gave outstanding protein resistance. The use of direct surface-initiated polymerization on surfaces resulting in covalently bound PG shows particular promise. ${ }^{34}$ Among the advantages of this surface-induced polymerization process is the resulting compatibility with a range of substrates including silicon oxide ${ }^{34}$ and amino- or alkoxy-modified surfaces. ${ }^{35}$ The nucleophilic groups are usually activated by anionic or cationic catalysis (e.g. sodium methoxide). The Terfort group 
optimized the reaction conditions by higher temperature and a suitable solvent. They showed that even catalyst-free ROP of glycidol allows the direct grafting of PG onto steel and other technically relevant materials such as silica and aluminum..$^{35,36}$ The dendritic coatings show excellent protein resistance when a thickness of $5 \mathrm{~nm}$ is exceeded. A bio-repulsion of $>99.9 \%$ against bacteria was reached for a polymer thickness above $17 \mathrm{~nm} \cdot{ }^{36}$

Due to the unique properties of dendritic PGs, we address the question of whether they are suitable for marine antifouling applications. These materials could be interesting hydrophilic candidates for incorporation into amphiphilic coatings. We synthesized PGs of different thicknesses, characterized them by state-of-the-art surface analysis and tested the coatings regarding their protein resistance. Laboratory diatom settlement, removal assays and field accumulation experiments under dynamic conditions were carried out to investigate their potential as FR materials. 


\section{Experimental Section}

\section{Preparation of polyglycerol-coated samples}

The PGs were prepared on glass or silicon wafers following previously published methods. ${ }^{35,36}$ To clean the substrates, they were sonicated in acetone (p.a.; VWR Chemicals, Radnor, USA) and Milli-Q Water (MQ, sterile and deionized water purified by a Siemens Water Technologies system) for three minutes and dried in a nitrogen flow. Nexterion $B \circledR$ Glass Slides (cleanroom quality, Schott, Mainz, Germany) were used as received. The samples were placed in an oxygen plasma (GaLa miniFlecto-PC-MFC; $0.4 \mathrm{mbar}, 80 \mathrm{~W}, 22 \mathrm{kHz}$ ) for $3 \mathrm{~min}$. The oxygen plasma removes organic impurities and enhances the native silicon oxide layer. Large numbers of slidesized samples $(75.6 \times 25.0 \mathrm{~mm})$ are required for statistical analysis in the assays for the biofouling tests. As conventional Teflon tubes are not available in the desired size range, we developed a reaction vessel made of Teflon specifically for the synthesis of PG (Figure 1B). The reaction vessel held eight Nexterion Glass Slides and two small silicon references. Reproducible samples could be obtained through internal temperature monitoring and gentle stirring. In order to prepare small silicon samples $\left(4 \mathrm{~cm}^{2}\right)$, which were used for surface analysis after the iterated ROP reinitiation synthesis and the protein assays, 20-ml disposable rolled-cuff brown glass bottles were used for the synthesis. The PG reaction vessel and rolled cuff bottles were heated overnight to $65^{\circ} \mathrm{C}$ to remove humidity and subsequently placed in a desiccator. A water- and oxygen-free environment was achieved by using the vacuum gas manifold technique. The hydroxylated samples were placed in the reaction vessel in $\mathrm{N}_{2}$ gas.

Solutions of 10-20 Vol\% glycidol (Sigma-Aldrich, St. Louis, USA; used as received) were prepared in N-methyl-2-pyrrolidon (VRW Chemicals, Radnor, USA) which had been previously dried with a mol sieve (4 $\AA$ ) and stored under inert gas. ${ }^{35,36}$ While applying a counter flow of $\mathrm{N}_{2}$ 
gas, the mixture was added to the reaction vessel so that the substrates were fully immersed into the solution. The vessels were closed, heated and kept at $140{ }^{\circ} \mathrm{C}$ for $2 \mathrm{~h}$. The synthesis was repeated with fresh solution until the desired thickness was reached. After synthesis, the surfaces were rinsed with acetone, and then subjected to sonication in acetone for a further $3 \mathrm{~min}$. Following this, the samples were sonicated in MQ for $3 \mathrm{~min}$, rinsed and dried in a nitrogen flow. The samples were stored under argon until used.

Preparation of control monolayers

Table 1. Summary of the surface analysis of the control surfaces used in the study. Six replicates per chemistry were used. The error bars represent the standard deviation.

\begin{tabular}{|lllll|}
\hline Label & Chemistry & Thickness & WCA & Substrate \\
& & $/ \mathrm{nm}$ & $/{ }^{\circ}$ & \\
\hline OTS & $\mathrm{SiCl}_{3}-\left(\mathrm{CH}_{2}\right)_{17}-\mathrm{CH}_{3}$ & $2.6 \pm 0.1$ & $110 \pm 3$ & Glass \\
DDT & $\mathrm{SH}-\left(\mathrm{CH}_{2}\right)_{11}-\mathrm{CH}_{3}$ & $1.1 \pm 0.1$ & $100 \pm 2$ & Gold \\
HUDT & $\mathrm{SH}-\left(\mathrm{CH}_{2}\right)_{11}-\mathrm{OH}$ & $1.1 \pm 0.1$ & $28 \pm 2$ & Gold \\
EG6OH & $\begin{array}{l}\mathrm{SH}-\left(\mathrm{CH}_{2}\right)_{2}-(\mathrm{O}- \\
\left.(\mathrm{CH})_{2}\right)_{6}-\mathrm{OH}\end{array}$ & $2.4 \pm 0.1$ & $31 \pm 1$ & Gold \\
& & & & \\
\hline
\end{tabular}

A set of control surfaces were prepared containing surfaces with well-known resistance against proteins and marine biofouling organisms to place the results from the PG coatings in context with other chemistries. The chemistries, water contact angles (WCAs) and film thicknesses are summarized in Table 1. Octadecylsilane (OTS)-coated glass slides were included as a hydrophobic control in the $N$. incerta assay. Some SAMs prepared from dodecane thiol (DDT), 11hydroxyundecanethiol (HUDT) and hydroxy-hexa(ethylene-glycol)-undecane thiol (EG6OH) on 
gold were included as controls for the rotating disk field test. The DDT was also used as a hydrophobic nonprotein-resistant control in the protein assays.

In order to coat the glass slides (Nexterion $B \circledR$, cleanroom quality, Schott, Mainz, Germany) and silicon substrates (Siegert Wafer, Aachen, Germany) with silanes, the substrates were first cleaned in an oxygen plasma before being transferred into a desiccator and thoroughly purged with nitrogen. A 0.5 M solution of octadecyltrichloro-silane (OTS; $\geq 90 \%$, Sigma-Aldrich, Radnor, USA) was prepared by adding the silane to a mixture of $75 \mathrm{Vol} \%$ cyclohexane $(\geq 99.98 \%)$ and 25 Vol\% chloroform ( $\geq 99.8 \%$; both Fisher Chemicals, Hampton, USA) at $\sim 5{ }^{\circ} \mathrm{C}$. The OTS mixture was added to the desiccator and sonicated (Bandelin Sonorex Super RK 106, 480 W) for 30 min while keeping the temperature below $10^{\circ} \mathrm{C}$. After silanization, the samples were removed from the desiccator, sonicated in cyclohexane and toluene for $3 \mathrm{~min}$ and dried in a nitrogen flow.

The SAMs were allowed to assemble in $1 \mathrm{mM}$ thiol solutions following protocols published previously. ${ }^{37}$ The DDT, HUDT and $\mathrm{EG}_{6} \mathrm{OH}$, all purchased from ProChimia Surfaces (Gdansk, Poland), were dissolved in ethanol p.a. (Roth, Germany). The gold-coated glass slides (Georg Albert, PVD, Silz, Germany) were cleaned under an ozone-generating UV-lamp for $1 \mathrm{~h}$ to remove surface contaminations. Subsequently, the samples were ultrasonicated in ethanol p.a., rinsed with ethanol p.a. and dried in a nitrogen flow. The samples were immersed for $24 \mathrm{~h}$ in thiol solution, rinsed with ethanol p.a., ultra-sonicated in ethanol p.a., rinsed again with ethanol p.a. and dried in a nitrogen flow for self-assembly.

\section{Spectroscopic Ellipsometry}

The film thickness was determined by spectroscopic ellipsometry. Measurements were carried out using a M-2000 V (J.A Woolam, Lincoln, USA) which measures in the spectral range between 
370 and $1000 \mathrm{~nm}$. All measurements were carried out at three different incidence angles $\left(65^{\circ}, 70^{\circ}\right.$ and $75^{\circ}$ ). The film on the surface was modeled as single organic layer with a wavelengthdependent refractive index described by a Cauchy model $(\mathrm{A}=1.45, \mathrm{~B}=0.01, \mathrm{C}=0)$. We used the software package CompleteEASE Version 4.98 for data analysis. Data obtained from at least two independent measurements on a minimum of two replicates per chemistry was averaged to determine the thickness. The error bars represent the standard deviation.

Contact angle goniometry

The static WCAs were measured using a custom-build goniometer. The water droplets $(\sim 50 \mu \mathrm{L})$ were applied on the sample surface and their shapes were recorded via a charge-coupled device camera. The implemented contour tracing algorithm automatically distinguished between drop and mirror image. The shape analysis was accomplished using Young's equation. The values presented were obtained from at least two positions measured on each of two replicate samples. Error bars represent the standard deviation.

ATR-FTIR

The ATR-FTIR spectra were obtained with a Bruker Tensor 27 spectrometer (Ettlingen, Germany) equipped with a liquid $\mathrm{N}_{2}$-cooled mercury cadmium telluride detector and a Ge-ATRcrystal (VariGATR, Harrick, USA). Prior to the measurement, the system was purged with nitrogen for $30 \mathrm{~min}$. The spectra represent the average of 150 scans.

Stability assay in aqueous media:

The stability of the three prepared coatings in aqueous solutions was tested by incubating the samples for defined durations of up to four weeks in MQ or salt water (SW). The SW was prepared by mixing the seven most abundant components of the artificial sea water (ASW) from the recipe described by Kester. ${ }^{38}$ Prior usage, the SW was filtered $(0.45 \mu \mathrm{m})$. We chose SW for the stability 
tests instead of filtered sea water to avoid any organic deposits that we observed in previous experiments. ${ }^{39}$ After incubation, the samples were rinsed thoroughly with MQ, dried in a nitrogen flow and analyzed by spectroscopic ellipsometry. Relative changes in the coating thicknesses were averaged and reported.

\section{Protein resistance assays}

Table 2. Molecular weight, isoelectric point and net charge under the $\mathrm{pH}$ of the buffered solution of the different proteins used for the adsorption assay.

\begin{tabular}{|llll|}
\hline Protein & Molecular weight & Isoelectric point & Charge \\
& $/ \mathrm{kDa}$ & & $/ \mathrm{pH} 7.4$ \\
\hline Fibrinogen & 340 & $\alpha 7.7$ & - \\
& & $\beta 8.7$ & \\
BSA & 66 & $\gamma 5.5$ & - \\
Lysozyme & 15 & 5.6 & + \\
$\begin{array}{l}\text { Pyruvate } \\
\text { kinase }\end{array}$ & 237 & 9.3 & + \\
\hline
\end{tabular}

The resistance of the PG coatings against the adsorption of fibrinogen from bovine plasma, bovine serum albumin (BSA), lysozyme from chicken egg white and pyruvate kinase (all from Sigma-Aldrich, used as received) was tested. The proteins were selected to cover a range of different sizes and net charges (Table 2). Fibrinogen and BSA carry a positive charge and lysozyme and pyruvate kinase a negative charge at $\mathrm{pH}$ 7.4. Fibrinogen and pyruvate kinase are larger proteins $(>200 \mathrm{kDa})$, while BSA and lysozyme are smaller $(<70 \mathrm{kDa})$. The PG-coated surfaces were preincubated in filtered $(0.45 \mu \mathrm{m})$ PBS buffer for $30 \mathrm{~min}$ for the protein resistance 
assays. After this preincubation step, the surfaces were gently rinsed with MQ and dried in a flow of $\mathrm{N}_{2}$ to determine the initial film thickness by spectroscopic ellipsometry. An amount of $1 \mathrm{mg} / \mathrm{ml}$ protein solutions of fibrinogen, BSA and lysozyme were prepared in filtered 1x PBS buffer (pH 7.4) for the protein resistance assay. A protein solution of $150 \mathrm{U} / \mathrm{ml}$ was used, corresponding to $0.3 \mathrm{mg} / \mathrm{ml}$ for pyruvate kinase. The samples were incubated in the protein solution for $30 \mathrm{~min}$ on a shaking table at $55 \mathrm{rpm}$. After incubation, the solutions containing the samples were diluted 100x with deionized water and removed from the solutions under a gentle MQ flow to avoid Langmuir layer formation. The protein layer thickness was determined by spectroscopic ellipsometry. In some cases, slightly negative protein film thicknesses were observed, which are probably due to slight variations in PG-coating thickness across the surface. The DDT was included as a nonprotein-resistant control. Laboratory diatom attachment and removal assay

The settlement and removal of cells of $N$. incerta were studied following published protocols. ${ }^{40}$ After three days of culturing, the cells were in log phase growth. Cells were washed three times in fresh ASW (Tropic Marin) before they were harvested and diluted to yield a suspension with a chlorophyll a content of approximately $0.25 \mu \mathrm{g} / \mathrm{ml}$. All coatings were pre-immersed for $30 \mathrm{~min}$ in $0.22 \mu \mathrm{m}$ filtered ASW prior to the assay. Cells were settled on three replicate-coated glass slides of each sample in individual quadriPERM dishes containing $10 \mathrm{ml}$ of suspension at $\sim 20^{\circ} \mathrm{C}$ on the laboratory bench. After $2 \mathrm{~h}$, the slides were placed on an orbital shaker for $5 \mathrm{~min}$ (60 rpm) followed by a submerged wash in ASW to remove cells which had not attached. Samples were fixed in $2.5 \mathrm{Vol} \%$ glutaraldehyde, air-dried and the density of cells attached to the surface was counted on each slide by eye, using a fluorescence microscope. Counts were made for 30 fields of view (each $0.15 \mathrm{~mm}^{2}$ ) on each slide. The removal of diatoms was tested with an additional set of three 
coated slides of each coating chemistry settled with cells of $N$. incerta, as described above. Slides with the attached cells were then exposed to a shear stress of $19 \mathrm{~Pa}$ in a turbulent flow water channel. ${ }^{41}$ Samples were fixed and the number of cells remaining attached were counted, as described above. The assay was repeated with two independent sets of samples at different times to verify the reproducibility of the results.

\section{Field assay using dynamic rotating disk}

The field test was carried out at the biofouling test site at Port Canaveral (Florida, USA) of the Florida Institute of Technology (FIT). We used a dynamic immersion setup described previously which is capable of creating well defined hydrodynamic conditions. ${ }^{42}$ The rotating disk setup was mounted on a floating raft to keep the immersion depth throughout the measurements constant at $0.6 \mathrm{~m}$. A constant rotation speed of $12 \mathrm{rpm}$ was applied to achieve a wall shear stress of $0.18 \mathrm{~Pa}$ and all surfaces were immersed for five days. The disk was unscrewed and lifted aboard the raft in a water-filled bucket without crossing the air-water interface for sample retrieval. Still under water, all samples were unscrewed from the disk and transferred into quadriPERM dishes (Sarstedt, Nümbrecht, Germany). The water-volume in the dishes was decreased to $5 \mathrm{~mL}$, and $5 \mathrm{~mL}$ of a $1 \mathrm{wt} \%$ glutaraldehyde solution (Thermo Fisher Scientific, Waltham, United States of America) solution was added carefully to obtain a final glutaraldehyde concentration of $0.5 \%$ for fixation. After 15 min, most of the fixative solution was removed without exposing the surfaces, followed by three washing steps with distilled water $(3 \mathrm{~min}$ each). Finally, the solution was removed and the samples were air-dried. Samples were subsequently analyzed by fluorescence microscopy (Eclipse Ti, Nikon Instruments, Japan) using a 20x magnification objective and a BV2A filter set (both Nikon Instruments). 
Each coating chemistry was tested with four replicates using glass slides (Nexterion B, $75.6 \times 25.0 \mathrm{~mm}$ ) and the assay was repeated with two independent sets to confirm reproducibility. Sixty fields of view, each $1.5 \times 1.5 \mathrm{~mm}$, were recorded automatically on every sample using bright field and fluorescence analysis (fluorescence light: ExFO XCite-121, Exceilias Technologies Corp, Germany; filter: BV-2A, EX: 400-440; DM: 455; BA: 470, Nikon Instruments, Japan) to obtain sufficient power for the statistical analysis. The location of the fields of view relative to the edges of the microscopic slides was the same on every sample. Bright field and fluorescence images were overlaid, and the diatoms present on the surface were manually counted using the ImageJ $1.51 \mathrm{j}$ software package. Manual counting was applied as the presence of other objects on the surface, such as sand, dirt, bacteria and diatom agglomerates (consisting of up to 1000 cells), hampered the automatic detection of the diatoms. During the counting, both the shape and the fluorescence were considered. A total of 240 fields of view were manually counted per sample type and outlier points detected and removed using the box plot method by John W. Tukey (1,5 x interquartile range (IQR), percentile: 25,75). A one-way ANOVA analysis with a post hoc Tukey test on log-transformed data was carried out to test the statistical significance $(\alpha=0.05)$ of the differences observed. ${ }^{43}$ 


\section{Results}

Sample preparation and characterization

In order to understand the surface induced polymerization behavior, the influence of pre-grafting thickness and monomer concentration was determined. Figure 2A shows the thickness obtained as a function of the PG starting thickness and concentration of glycidol in solution for a $2 \mathrm{~h}$ polymerization reaction. The data shows that the film thickness obtained increased nearly linearly with increasing primer PG layer thickness with a slope that depended on the monomer concentration. Linear fits were applied to estimate the primer thickness required for a target PG thickness, and the corresponding parameters are shown in Figure 2B. In particular, at higher glycidol concentrations, the presence of an initial PG film enhanced the polymerization rate. Based on this analysis, the desired layer thickness can readily be adjusted. As the linearity of the slopes in Figure 2A was higher for $10 \%$ and $15 \%$ solutions $\left(\mathrm{R}^{2}\right.$ values in Figure $\left.2 \mathrm{~B}\right)$, these conditions were mainly selected for the preparation of the surfaces used in this study. We found that multiple subsequent and independent, $2 \mathrm{~h}$ lasting polymerization reactions in replenished $10-15 \%$ monomer solution was best suited to reproducibly reach the desired coating thickness. The protocol developed for iterated ROP reinitiation synthesis allowed us to prepare the desired PG thickness on a batch of glass slides with sufficient surface areas for biological testing. 


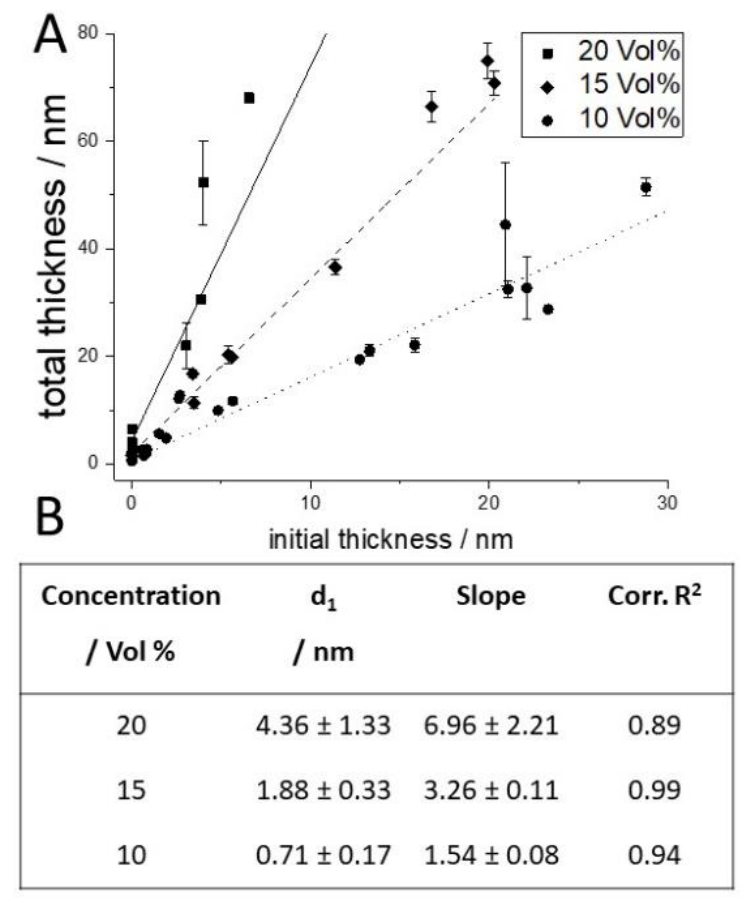

Figure 2. A: Iterated ROP reinitiation synthesis $(2 \mathrm{~h}$ reaction time) at three different concentrations of the glycidol monomer. The error bars represent the standard deviation of two measurements on each of the four replicates. B: Summary of the parameters, which were obtained from the linear regression of the relationship of PG thickness and initial thickness. Thickness $\mathrm{d}_{1}$ was obtained by spectroscopic ellipsometry after the first polymerization step from the pristine surface. Slope

and corrected $\mathrm{R}^{2}$ are obtained by linear regression fits.
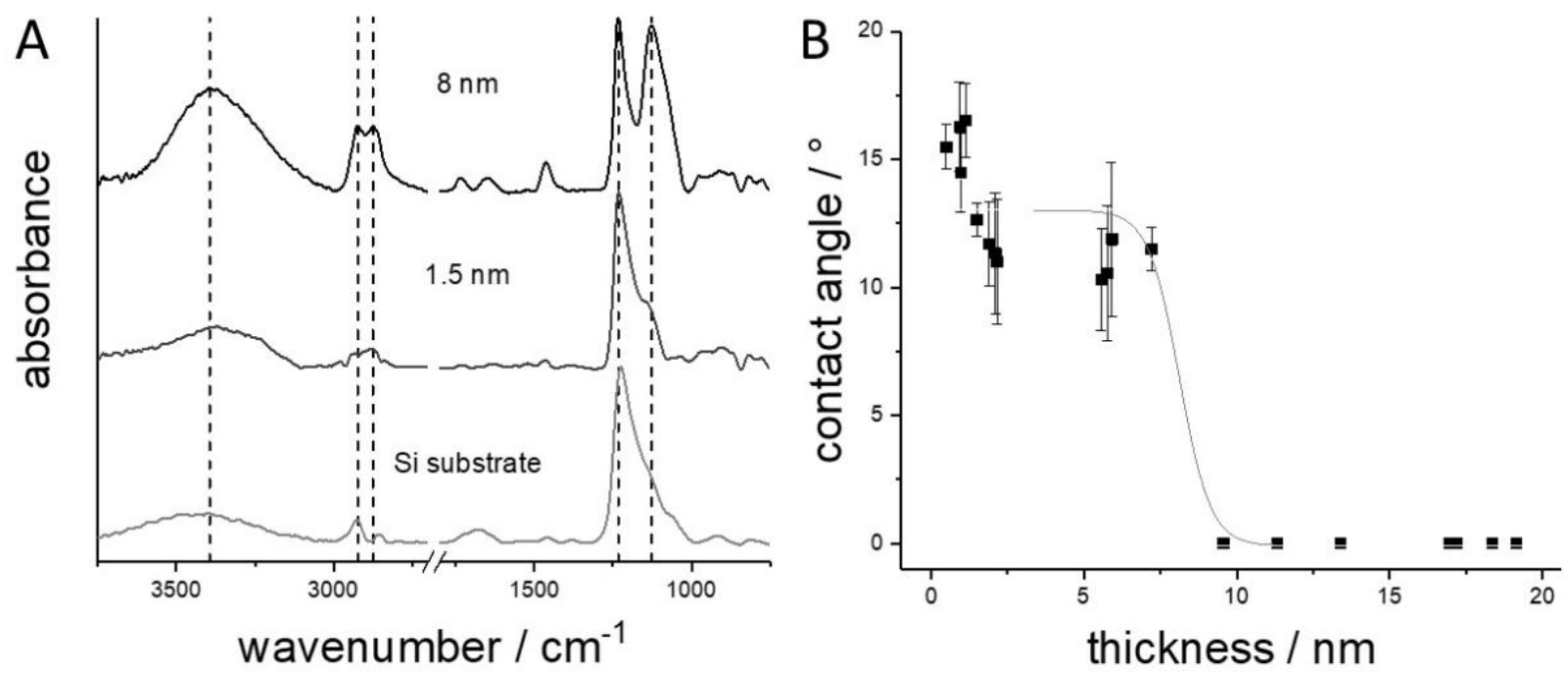

Figure 3. A: ATR-FTIR spectra of two different thicknesses of PG $(8 \mathrm{~nm}$ and $1.5 \mathrm{~nm})$ and a blank silicon wafer as a control. Three vibrational modes indicate formation of the PG, which are the ether stretch vibrations at $1127 \mathrm{~cm}^{-1}, \mathrm{C}-\mathrm{H}$ stretch vibrations at $2923 \mathrm{~cm}^{-1}$ and $2875 \mathrm{~cm}^{-1}$, and 
$\mathrm{OH}$ stretch vibration around $3400 \mathrm{~cm}^{-1}$. These signals increase in intensity with increasing polymer thickness. B: Static water contact angle (WCA) of PG at different film thicknesses. At around $8 \mathrm{~nm}$, the WCA shows a drop from $12^{\circ}$ to less than $5^{\circ}$. The error bars represent the standard deviation of two measurements on each of the two replicates.

ATR-FTIR spectroscopy was applied to verify the formation of PG layers on the surfaces (Figure 3A). The bottom spectrum shows the uncoated and untreated silicon substrate which was dominated by the $v(\mathrm{Si}-\mathrm{O}-\mathrm{Si})$ stretch vibration at $1233 \mathrm{~cm}^{-1}$ and a small $(\mathrm{H}-\mathrm{O}-\mathrm{H})$ bending vibration of free molecular water around 1630 to $1760 \mathrm{~cm}^{-1} .{ }^{44}$ The middle and top spectra show PG films with a thickness of 1.5 and $8 \mathrm{~nm}$, respectively. The $v(\mathrm{C}-\mathrm{H})$ stretching modes between 2800 and $3000 \mathrm{~cm}^{-1}$ increased with larger PG thickness and appeared as a double peak at 2923 and $2875 \mathrm{~cm}^{-1} \cdot{ }^{36}$ Such a signal increase is also seen in the case of the $(\mathrm{C}-\mathrm{H})$ bending mode at $1460 \mathrm{~cm}^{-1}$ and the $\mathrm{OH}$ stretch vibration region at approximately $3400 \mathrm{~cm}^{-1}$. The (C-OC) stretch vibration at $1127 \mathrm{~cm}^{-1}$ was highly characteristic of the ether groups in the PG coating. It appears as a shoulder next to the silicon peak for thin coatings and develops into a separated peak at higher film thickness.

The wettability of the coatings was determined by static WCA goniometry. The dependence of the WCA on the film thickness is depicted in Figure 3B. The pure silicon substrate had a WCA of around $35^{\circ}$. Even very small thicknesses of PG decreased the WCA to values around $10-15^{\circ}$. There is a transition of the WCA to nearly $0^{\circ}$ at $8 \mathrm{~nm}$, which means that a fully wetted surface was accomplished. 


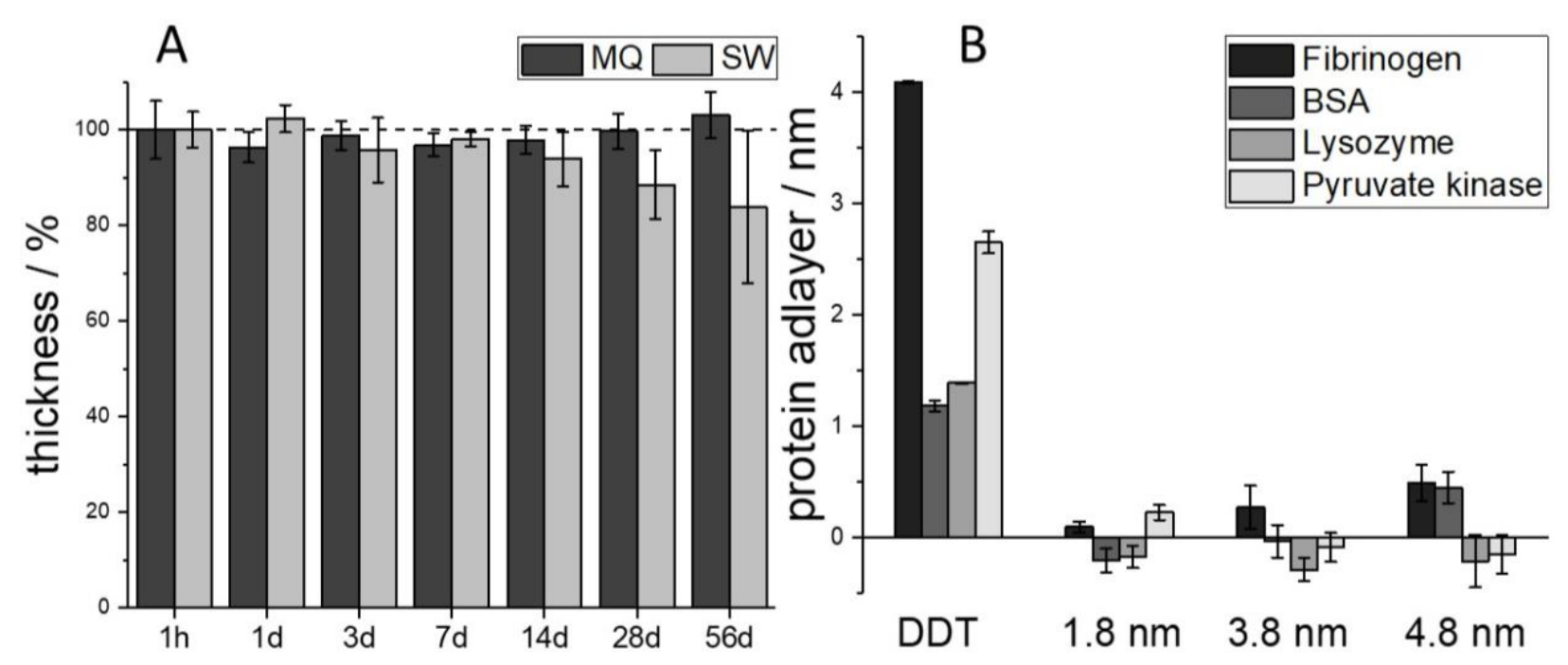

Figure 4. A: Stability test of PG coatings on silica in MQ and SW over two months (h, hour; d, day). Data are represented as relative change in coating thickness over time as determined by spectroscopic ellipsometry. The error bars represent the standard deviation determined from two independent thickness measurements on each of three replicate surfaces. The dashed line indicates the initial film thickness (100\% mark). B: Protein assay with four different proteins. A DDT-SAM was included in the assay as negative control. PG coatings are, with a few exceptions, resistant against protein adsorption. The error bars represent the standard deviation from three independent measurements on each of the three replicates.

The stability of the PG coatings in Milli-Q water (MQ) and salt water (SW) was tested over two months (Figure 4A). After immersion in the SW solution for the given time, samples were rinsed with MQ water to remove any excess salt from the surface prior to the spectroscopic ellipsometry measurements. A one-way ANOVA analysis (significance level of $\alpha=0.05$ ) showed that there was no significant difference in coating thickness over a 56-day period of immersion in MQ. The 
stability was slightly reduced in SW, but more than $80 \%$ of the film thickness remained on the surfaces even after an exposure of two months.

The resistance of the PG films prepared against adsorption of proteins was determined against fibrinogen, BSA, lysozyme and pyruvate kinase. The four proteins were chosen due to their different size and net charge. As shown in Figure 4B, all PG coatings were able to strongly reduce the attachment of the different proteins regarding the nonprotein-resistant DDT control. The negative values are probably due to slight thickness variations across the coatings as measurements were recorded at arbitrary positions on the surfaces.

Laboratory diatom attachment and removal assay
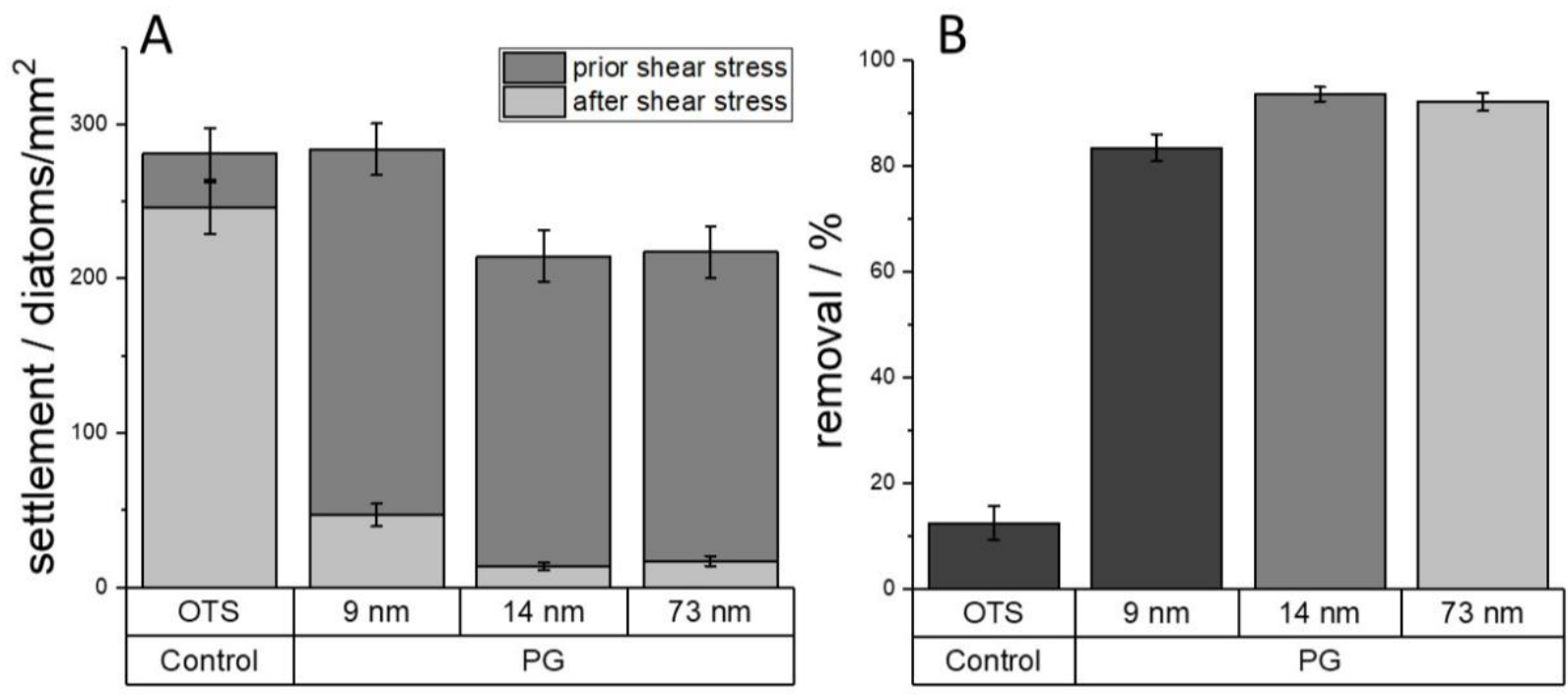

Figure 5. A: The density of settled diatoms on the controls and PG surfaces after $2 \mathrm{~h}$. The bright parts of the bars indicate the density of diatoms remaining on PG surfaces after exposure to a shear stress of $19 \mathrm{~Pa}$. Each data point is the mean from 90 counts on three replicate slides. Error bars show the $95 \%$ confidence limits. B: Percentage removal of diatoms from PG surfaces by the 
turbulent flow with a wall shear stress of $19 \mathrm{~Pa}$. Each bar is the mean of 90 counts on three replicate slides. Error bars show $95 \%$ confidence limits derived from arcsine transformed data.

The same number of cells were available for adhesion to each surface in the attachment assay. Unattached cells can be removed in the washing procedure. The results indicate that similar numbers of cells were able to form an initial attachment on each surface and stayed adhered after the washing stage (Figure 5A). A one-way ANOVA with post hoc Tukey test (significance level of $\alpha=0.05$ ) indicates two groups which showed statistically significant differences. The OTS and the $9 \mathrm{~nm}$ PG coating were significantly different to the 14 and $73 \mathrm{~nm}$ PG. The surfaces could not be distinguished within these groups. After exposure to a shear stress of $19 \mathrm{~Pa}$, the diatom density on the PGs was reduced more than on the OTS control surfaces.

The lower portion of each bar in (A) shows the density of the diatoms remaining and panel (B) presents the fraction of diatoms removed after exposure to $19 \mathrm{~Pa}$ wall shear stress. More than 92 $\%$ of diatom density was reduced on the two thickest PG coatings (Figure 5B). The OTS, as a negative standard, showed only $12 \%$ removal. The removal was significantly different within all samples tested, except for the two thickest PG coatings $(\mathrm{p}$-value $=0.999)$. 

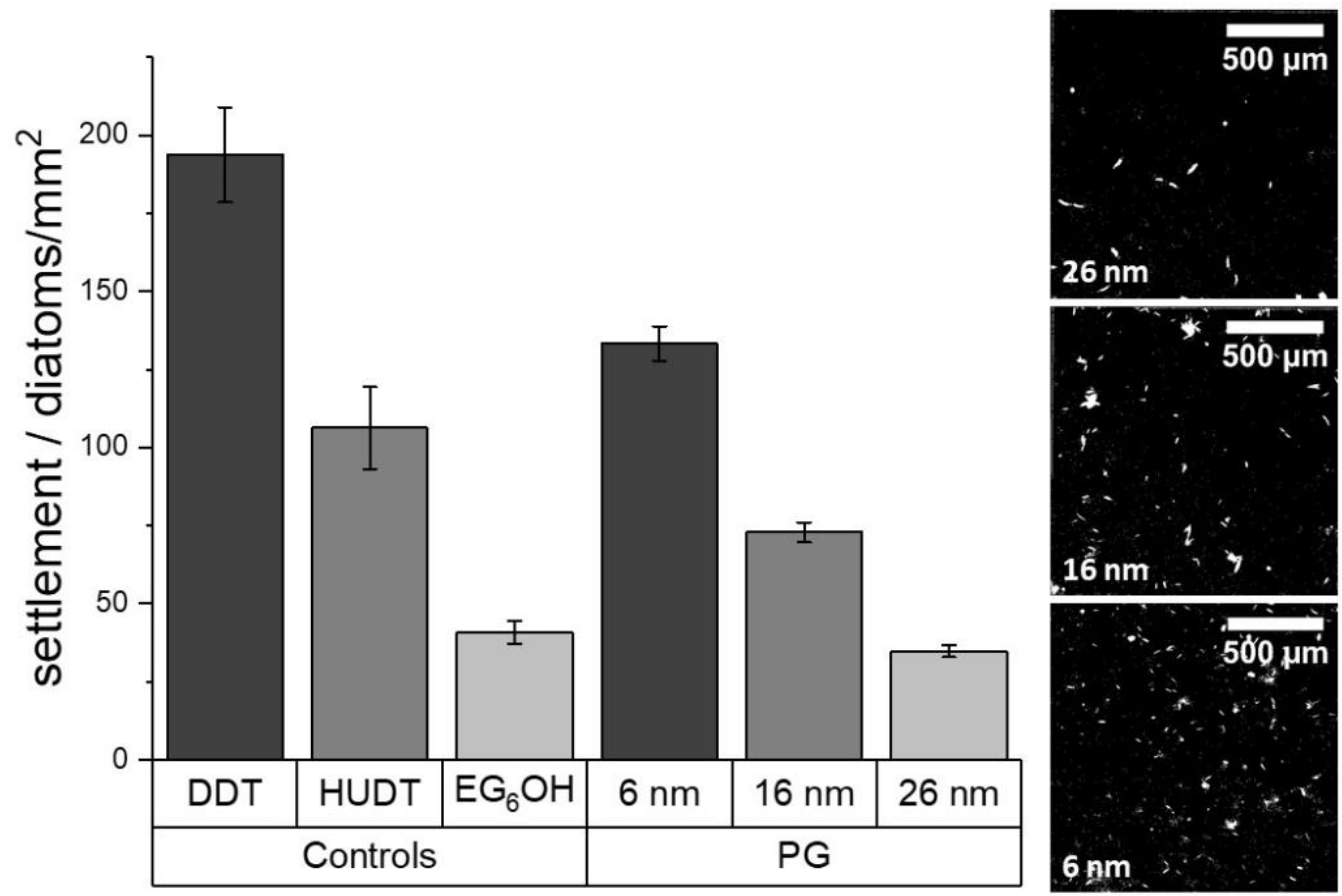

Figure 6. Comparison of the performance of the three SAM controls (DDT, HUDT, EG6OH) and three thicknesses of PG in the dynamic field test in Florida. Each data point is the average of 240 counts of diatoms on four replicate slides. Error bars represent the standard error of the mean.

Figure 6 shows the average surface density of diatoms of the standard SAMs (controls), the PGs with different thicknesses and three representative microscopy images of the PGs after immersion for five days in the ocean at the Port Canaveral test site using a dynamic rotating disk setup. The wall shear stress acting on the samples during the field immersion test was $0.18 \mathrm{~Pa}$. After fixation of the slides, diatom densities were manually counted using bright field and fluorescence analysis. The bar graph shows one of two sets immersed at different time points, each containing four replicates per chemistry. In addition to the PGs, a set of controls published previously with known FR properties, including hydrophobic DDT, hydroxylated HUDT and linear ethylene glycol $\mathrm{EG}_{6} \mathrm{OH},{ }^{42}$ that were exposed during the same experiment are shown in Figure 6. The DDT showed 
high numbers of diatoms, while there were lower diatom densities on the hydrophilic HUDT and $\mathrm{EG}_{6} \mathrm{OH}$. The number of diatoms on the PG coatings decreased with increasing PG film thickness. The thickest PG layer (26 nm) performed slightly but not significantly better ( $35 \pm 2$ diatoms $/ \mathrm{mm}^{2}$ ) than $\mathrm{EG}_{6} \mathrm{OH}\left(41 \pm 4\right.$ diatoms $\left./ \mathrm{mm}^{2}\right)$. The $16 \mathrm{~nm}$ PG layer $\left(73 \pm 3\right.$ diatoms $\left./ \mathrm{mm}^{2}\right)$ showed intermediate densities between $\mathrm{EG}_{6} \mathrm{OH}$ and HUDT $\left(106 \pm 13\right.$ diatoms $\left./ \mathrm{mm}^{2}\right)$. The lowest PG thickness of $6 \mathrm{~nm}\left(133 \pm 6\right.$ diatoms $\left./ \mathrm{mm}^{2}\right)$ outperformed DDT $\left(194 \pm 15\right.$ diatoms $\left./ \mathrm{mm}^{2}\right)$. All surfaces were significantly different except for the comparisons between HUDT with $16 \mathrm{~nm}$ PG and $\mathrm{EG}_{6} \mathrm{OH}$ with $26 \mathrm{~nm}$ PG (ANOVA with post hoc Tukey test, $\alpha=0.05$ ). The p-value for the comparison of HUDT with $6 \mathrm{~nm}$ PG was 0.804 and for $\mathrm{EG}_{6} \mathrm{OH}$ with $26 \mathrm{~nm}$ PG 0.995, which indicates a high conformity. 


\section{Discussion}

A dedicated Teflon reaction vessel was developed to reproducibly prepare PG coatings on multiple glass slides in parallel. The thermally-induced, catalyst-free, iterated ROP reinitiation synthesis has proven to be a facile method of obtaining reproducible, high quality coatings on a large number of glass slides. A sufficient number of slides could be prepared using the reaction vessel that provided the total surface area desired for the biological tests. We restricted the synthesis time and reinitiated the reaction as the PG synthesis is very sensitive to traces of water which causes hydrolysis of glycidol and a range of side reactions. Despite the dry solvents used, the large number of samples in the Teflon reaction vessel made it difficult to obtain a perfectly dry environment. Thus, shorter reaction times with a reinitiation step enhanced the reproducibility of the results.

The PG films showed a superior stability in both MQ and SW environments for more than two months, which indicates that the chemistry is well suited for technical applications. The coatings showed excellent protein resistance and reduced adsorption significantly compared to the nonprotein-resistant DDT controls. The results are in line with literature reports about PG and several PG derivatives that showed strong reduction of fibrinogen and albumin adsorption. . $^{31,32,35,36}$ The current results, including those for pyruvate kinase and lysozyme also agreed with these previous reports. Even thicknesses below the $5 \mathrm{~nm}$ reported were sufficient to produce highly protein-resistant surfaces. Similar results were previously reported for linear PEG-SAMs and oligoethylene glycol SAMs down to three units ( $\mathrm{EG}_{3} \mathrm{OH}, 1.8 \mathrm{~nm}$ thickness) which were completely resistant to fibrinogen adsorption..$^{45,46}$

As an earlier article showed a good resistance of the PG coatings to freshwater bacteria, ${ }^{35}$ we explored whether such coatings show antifouling properties against marine diatoms, which are a 
challenge for many commercial FR coatings. ${ }^{47-49}$ Relatively high diatom numbers settled on the dendritic PGs and similar initial attachment was observed on the controls in the static settlement tests. After exposure to a hydrodynamic shear stress (19 Pa), nearly all diatoms remained attached on the OTS controls, $\left(\mathrm{WCA}=110^{\circ}\right)$, while $58 \%$ were removed on the more hydrophilic glass surfaces $\left(\mathrm{WCA}=30^{\circ}\right.$ ). The PGs showed an exceptional FR performance with removal of up to 94 $\%$ of the cells. The good FR performance of dendritic PGs is not unexpected as ethylene glycols have been found in the past to successfully suppress attachment of marine bacteria. ${ }^{50}$ It has also been shown that the marine antifouling behavior of ethylene glycols depends strongly on both their termination and chain length. ${ }^{45,51}$ Hydrophilic, linear oligoethylene glycols were particularly able to inhibit the settlement of $N$. incerta. After application of a shear stress, the number of cells remaining was low both on PEGs and EGs. While the weak attachment on the ethylene glycols is in excellent agreement with the superior FR properties found on the PGs, the high initial settlement on the PGs has yet to be understood. The thicker PG layers $(73 \mathrm{~nm})$ were more effective in releasing diatoms than the thinner $(9 \mathrm{~nm})$ ones. This is surprising considering that even the $9-\mathrm{nm}$ thick coatings are thick compared to oligoethylene/polyethylene glycol SAMs which had thicknesses $<3 \mathrm{~nm} .{ }^{45}$ The PGs seem to require a higher thickness to become resistant compared to the linear EGs. One could speculate that the dendritic structure of the polymers contributes to this effect. A comparison to literature is difficult as the number of studies that use hyperbranched polymers in marine antifouling research is still limited. One of the prominent examples is PEG containing HBFP. ${ }^{10}$ These polymers are more hydrophobic than our dendritic PGs and also showed relatively high initial settlement. In agreement with our results, particularly the PEG-rich coatings, showed very good fouling release properties ( $65 \%$ diatom removal). 
Testing model chemistries such as the thin-film PG coatings in the field is extremely challenging. Static immersion frequently leads to only minor shifts in diatom population on surfaces. ${ }^{37} \mathrm{We}$ applied our recently developed dynamic field exposure assay based on a rotating disk capable of distinguishing early biofilm formation on model surfaces. ${ }^{42}$ The rotating disk has a speed of $12 \mathrm{rpm}$, which results in a wall shear stress of $0.18 \mathrm{~Pa}$, a value that was found to be suitable for discriminating between the performance of the different surface chemistries regarding diatom attachment under dynamic conditions in the laboratory. ${ }^{52}$ The dynamic exposure in the ocean environment showed differences in diatom attachment on a PG coating 16 and $26 \mathrm{~nm}$ thick. Compared to the laboratory assay, the field test showed that fouling resistance was greatly enhanced as the thickness of the PG layer increased. 


\section{Conclusion}

The challenge of preparing reproducible batches of PG-coated glass slides via ROP was tackled using a tailored reaction vessel and iterated ROP reinitiation synthesis. The ROP polymerization was thermally induced, worked free of catalyst and yielded high quality PG layers. We demonstrated that the PG coatings were highly protein-resistant and stable in both MQ and SW. While diatom settlement was not inhibited, adhesion strength was low, and the cells were easily removed in a turbulent flow water channel. A removal up to $94 \%$ was achieved even at moderate wall shear stresses of $19 \mathrm{~Pa}$. The laboratory data was supported by field tests in which low diatom attachment under dynamic conditions was found. As a general trend, protein resistance and fouling-release properties were enhanced at higher coating thicknesses. In view of the high stability of the PGs in the marine environment and considering the fact that they are less prone to oxidative degradation than PEG materials, these surfaces have potential for inclusion in the next generation of fouling-release coatings. 


\section{Associated Content}

Supporting Information List:

1. Results of second laboratory assay with Navicula incerta.

2. Comparison of the statistical significance of the discrimination of the PG-samples and controls in the settlement and removal of diatoms in Navicula incerta by post hoc Tukey test for the assay which is shown in the publication.

3. Comparison of the statistical significance of the differences among the PG-samples and controls in the settlement of diatoms in field tests by post hoc tukey analysis after logtransformation of the data from the assay which was shown in the main publication.

\section{Author Information}

* Axel Rosenhahn, Analytical Chemistry - Biointerfaces, Ruhr-University Bochum, 44780

Bochum, Germany

ORCID:

Robin Wanka: 0000-0001-8892-9399

Axel Rosenhahn: 0000-0001-9393-7190

\section{Author Contributions}

The manuscript was written through contributions of all authors. All authors have given approval to the final version of the manuscript.

\section{Funding Sources}

ONR N00014-16-12979 (RUB), ONR N00014-16-1-3123 (FIT), ONR N00014-16-1-2988

(ASC), N00014-16-1-3125 (ASC and JAF), DFG RO2524/4-1 and Mercur Pr-2015-0018. 


\section{Acknowledgements}

The work was funded by ONR N00014-16-12979 (RUB), ONR N00014-16-1-3123 (FIT), ONR N00014-16-1-2988 (ASC), N00014-16-1-3125 (ASC and JAF), DFG RO2524/4-1 and Mercur Pr2015-0018. The authors thank Ajitha Amuthalingam for preparation of the OTS controls, Kevin Profita for exploring the iterated ROP reinitiation synthesis, Sebastian Kursawa for assistance in counting algae and Armin Lindner from the mechanical workshop of our faculty at the RUB for his technical support in the design and fabrication of the Teflon box. 


\section{References}

(1) Rosenhahn, A.; Ederth, T.; Pettitt, M. E. Advanced nanostructures for the control of biofouling: The FP6 EU Integrated Project AMBIO. Biointerphases 2008, 3, IR1-5.

(2) Schultz, M. P.; Bendick, J. A.; Holm, E. R.; Hertel, W. M. Economic impact of biofouling on a naval surface ship. Biofouling 2011, 27, 87-98.

(3) Kirschner, C. M.; Brennan, A. B. Bio-Inspired Antifouling Strategies. Annu. Rev. Mater. Res. 2012, 42, 211-229.

(4) Callow, J. A.; Callow, M. E. Trends in the development of environmentally friendly fouling-resistant marine coatings. Nat. Commun. 2011, 2, 244.

(5) Ratner, B. D.; Bryant, S. J. Biomaterials: Where We Have Been and Where We Are Going. Annu. Rev. Biomed. Eng. 2004, 6, 41-75.

(6) Gorbet, M. B.; Sefton, M. V. Biomaterial-associated thrombosis: Roles of coagulation factors, complement, platelets and leukocytes. Biomaterials 2004, 25, 5681-5703.

(7) Finlay, J. A.; Krishnan, S.; Callow, M. E.; Callow, J. A.; Dong, R.; Asgill, N.; Wong, K.; Kramer, E. J.; Ober, C. K. Settlement of Ulva zoospores on patterned fluorinated and PEGylated monolayer surfaces. Langmuir 2008, 24, 503-510.

(8) Blaszykowski, C.; Sheikh, S.; Thompson, M. Surface chemistry to minimize fouling from blood-based fluids. Chem. Soc. Rev. 2012, 41, 5599.

(9) Rosenhahn, A.; Schilp, S.; Kreuzer, H. J.; Grunze, M. The role of "inert" surface chemistry in marine biofouling prevention. Phys. Chem. Chem. Phys. 2010, 12, 4275-4286.

(10) Imbesi, P. M., Finlay, J. A., Aldred, N., Eller, M. J., Felder, S. E., Pollack, K. A.; Lonnecker, A. T.; Raymond, J. E.; Mackay, M. E.; Schweikert, E. A.; Clare, A. S.; Callow, J. A.; Callow, M. E.; Wooley, K. L. Targeted surface nanocomplexity: Two-dimensional control over the composition, physical properties and anti-biofouling performance of hyperbranched fluoropolymer-poly(ethylene glycol) amphiphilic crosslinked networks. Polym. Chem. 2012, 3, 3121.

(11) Bartels, J. W.; Cheng, C.; Powell, K. T.; Xu, J.; Wooley, K. L. Hyperbranched Fluoropolymers and their Hybridization into Complex Amphiphilic Crosslinked Copolymer Networks. Macromol. Chem. Phys. 2007, 208, 1676-1687.

(12) Lejars, M.; Margaillan, A.; Bressy, C. Fouling release coatings: A nontoxic alternative to biocidal antifouling coatings. Chem. Rev. 2012, 112, 4347-4390.

(13) Zhou, Z.; Calabrese, D. R.; Taylor, W.; Finlay, J. A.; Callow, M. E.; Callow, J. A.; Fischer, D.; Kramer, E. J.; Ober, C. K. Amphiphilic triblock copolymers with PEGylated hydrocarbon structures as environmentally friendly marine antifouling and fouling-release coatings. Biofouling 2014, 30, 589-604.

(14) Gan, D.; Mueller, A.; Wooley, K. L. Amphiphilic and hydrophobic surface patterns generated from hyperbranched fluoropolymer/linear polymer networks: Minimally adhesive coatings via the crosslinking of hyperbranched fluoropolymers. J. Polym. Sci. A Polym. Chem. 2003, 41, 3531-3540.

(15) Imbesi, P. M.; Raymond, J. E.; Tucker, B. S.; Wooley, K. L. Thiol-ene "click" networks from amphiphilic fluoropolymers: Full synthesis and characterization of a benchmark anti-biofouling surface. J. Mater. Chem. 2012, 22, 19462.

(16) Pollack, K. A.; Imbesi, P. M.; Raymond, J. E.; Wooley, K. L. Hyperbranched fluoropolymerpolydimethylsiloxane-poly(ethylene glycol) cross-linked terpolymer networks designed for marine and biomedical applications: Heterogeneous nontoxic antibiofouling surfaces. ACS Appl. Mater. Interfaces 2014, 6, 19265-19274. 
(17) Imbesi, P. M.; Gohad, N. V.; Eller, M. J.; Orihuela, B.; Rittschof, D.; Schweikert, E. A.; Mount, A. S.; Wooley, K. L. Noradrenaline-functionalized hyperbranched fluoropolymer-poly(ethylene glycol) crosslinked networks as dual-mode, anti-biofouling coatings. ACS nano 2012, 6, 1503-1512.

(18) Park, D.; Weinman, C. J.; Finlay, J. A.; Fletcher, B. R.; Pait, M. Y.; Sundaram, H. S.; Dimitriou, M. D.; Sohn, K. E.; Callow, M. E.; Callow, J. A.; Handlin, D. L.; Willis, C. L.; Fischer, D. A.; Kramer, E. J.; Ober, C. K. Amphiphilic surface active triblock copolymers with mixed hydrophobic and hydrophilic side chains for tuned marine fouling-release properties. Langmuir 2010, 26, 9772-9781.

(19) Nelson, C. M.; Raghavan, S.; Tan, J. L.; Chen, C. S. Degradation of Micropatterned Surfaces by CellDependent and -Independent Processes †. Langmuir 2003, 19, 1493-1499.

(20) Luk, Y.-Y.; Kato, M.; Mrksich, M. Self-Assembled Monolayers of Alkanethiolates Presenting Mannitol Groups Are Inert to Protein Adsorption and Cell Attachment. Langmuir 2000, 16, 9604-9608.

(21) Sharma, S.; Johnson, R. W.; Desai, T. A. Evaluation of the Stability of Nonfouling Ultrathin Poly(ethylene glycol) Films for Silicon-Based Microdevices. Langmuir 2004, 20, 348-356.

(22) Jeyachandran, Y. L.; Terfort, A.; Zharnikov, M. Controlled Modification of Protein-Repelling SelfAssembled Monolayers by Ultraviolet Light: The Effect of the Wavelength. J. Phys. Chem. C 2012, 116, 9019-9028.

(23) Jeyachandran, Y. L.; Weber, T.; Terfort, A.; Zharnikov, M. Application of Long Wavelength Ultraviolet Radiation for Modification and Patterning of Protein-Repelling Monolayers. J. Phys. Chem. C 2013, 117, 5824-5830.

(24) Frey, H.; Haag, R. Dendritic polyglycerol: A new versatile biocompatible material. Reviews in Molecular Biotechnology 2002, 90, 257-267.

(25) Kainthan, R. K.; Janzen, J.; Levin, E.; Devine, D. V.; Brooks, D. E. Biocompatibility testing of branched and linear polyglycidol. Biomacromolecules 2006, 7, 703-709.

(26) Irvine, D. J.; Mayes, A. M.; Griffith-Cima, L. Self-Consistent Field Analysis of Grafted Star Polymers. Macromolecules 1996, 29, 6037-6043.

(27) Groll, J.; Ameringer, T.; Spatz, J. P.; Moeller, M. Ultrathin coatings from isocyanate-terminated star PEG prepolymers: Layer formation and characterization. Langmuir 2005, 21, 1991-1999.

(28) Groll, J.; Fiedler, J.; Engelhard, E.; Ameringer, T.; Tugulu, S.; Klok, H.-A.; Brenner, R. E.; Moeller, M. A novel star PEG-derived surface coating for specific cell adhesion. J. Biomed. Mater Res. 2005, 74, 607617.

(29) Meyerbröker, N.; Kriesche, T.; Zharnikov, M. Novel ultrathin poly(ethylene glycol) films as flexible platform for biological applications and plasmonics. ACS applied materials \& interfaces 2013, 5, $2641-$ 2649.

(30) Gasteier, P.; Reska, A.; Schulte, P.; Salber, J.; Offenhäusser, A.; Moeller, M.; Groll, J. Surface grafting of PEO-based star-shaped molecules for bioanalytical and biomedical applications. Macromol. Biosci.

2007, 7, 1010-1023.

(31) Siegers, C.; Biesalski, M.; Haag, R. Self-assembled monolayers of dendritic polyglycerol derivatives on gold that resist the adsorption of proteins. Chemisty 2004, 10, 2831-2838.

(32) Wyszogrodzka, M.; Haag, R. Synthesis and characterization of glycerol dendrons, self-assembled monolayers on gold: A detailed study of their protein resistance. Biomacromolecules 2009, 10, 10431054.

(33) Weinhart, M.; Becherer, T.; Schnurbusch, N.; Schwibbert, K.; Kunte, H.-J.; Haag, R. Linear and Hyperbranched Polyglycerol Derivatives as Excellent Bioinert Glass Coating Materials. Adv. Eng. Mater. 2011, 13, B501-B510. 
(34) Khan, M.; Huck, W. T. S. Hyperbranched Polyglycidol on Si/SiO 2 Surfaces via Surface-Initiated Polymerization. Macromolecules 2003, 36, 5088-5093.

(35) Weber, T.; Gies, Y.; Terfort, A. Bacteria-Repulsive Polyglycerol Surfaces by Grafting Polymerization onto Aminopropylated Surfaces. Langmuir 2012, 28, 15916-15921.

(36) Weber, T.; Bechthold, M.; Winkler, T.; Dauselt, J.; Terfort, A. Direct grafting of anti-fouling polyglycerol layers to steel and other technically relevant materials. Colloids and Surf. B: Biointerfaces 2013, 111, 360-366.

(37) Thome, I.; Bauer, S.; Vater, S.; Zargiel, K.; Finlay, J. A.; Arpa-Sancet, M. P.; Alles, M.; Callow, J. A.; Callow, M. E.; Swain, G. W. Grunze, M.; Rosenhahn, A. Conditioning of self-assembled monolayers at two static immersion test sites along the east coast of Florida and its effect on early fouling development. Biofouling 2014, 30, 1011-1021.

(38) Kester, D. R.; Duedall, I. W.; Connors, D. N.; Pytkowicz, R. M. Preparation of artificial seawater. Limnol. Oceangr. 1967, 12, 176-179.

(39) Thome, I.; Pettitt, M. E.; Callow, M. E.; Callow, J. A.; Grunze, M.; Rosenhahn, A. Conditioning of surfaces by macromolecules and its implication for the settlement of zoospores of the green alga Ulva linza. Biofouling 2012, 28, 501-510.

(40) Finlay, J. A.; Bennett, S. M.; Brewer, L. H.; Sokolova, A.; Clay, G.; Gunari, N.; Meyer, A. E.; Walker, G. C.; Wendt, D. E.; Callow, M. E.; Callow, J. A.; Detty, M. R. Barnacle settlement and the adhesion of protein and diatom microfouling to xerogel films with varying surface energy and water wettability. Biofouling 2010, 26, 657-666.

(41) Schultz, M. P.; Finlay, J. A.; Callow, M. E.; Callow, J. A. A turbulent channel flow apparatus for the determination of the adhesion strength of microfouling organisms. Biofouling 2000, 15, 243-251.

(42) Nolte, K. A.; Koc, J.; Barros, J. M.; Hunsucker, K.; Schultz, M. P.; Swain, G. E.; Rosenhahn, A. Dynamic field testing of coating chemistry candidates by a rotating disk system. Biofouling 2018, 49, 1-12.

(43) Tukey, J. W. Exploratory Data Analysis; Addison-Wesley series in behavioral science Quantitative methods; Addison-Wesley: Reading, Mass., 1997.

(44) Ben Slama, S.; Hajji, M.; Ezzaouia, H. Crystallization of amorphous silicon thin films deposited by PECVD on nickel-metalized porous silicon. Nanoscale Res. Lett. 2012, 7, 464.

(45) Schilp, S.; Rosenhahn, A.; Pettitt, M. E.; Bowen, J.; Callow, M. E.; Callow, J. A.; Grunze, M. Physicochemical properties of (ethylene glycol)-containing self-assembled monolayers relevant for protein and algal cell resistance. Langmuir 2009, 25, 10077-10082.

(46) Herrwerth, S.; Eck, W.; Reinhardt, S.; Grunze, M. Factors that determine the protein resistance of oligoether self-assembled monolayers --internal hydrophilicity, terminal hydrophilicity, and lateral packing density. JACS 2003, 125, 9359-9366.

(47) Hunsucker, K. Z.; Koka, A.; Lund, G.; Swain, G. Diatom community structure on in-service cruise ship hulls. Biofouling 2014, 30, 1133-1140.

(48) Holland, R.; Dugdale, T. M.; Wetherbee, R.; Brennan, A. B.; Finlay, J. A.; Callow, J. A.; Callow, M. E. Adhesion and motility of fouling diatoms on a silicone elastomer. Biofouling 2004, 20, 323-329.

(49) Finlay, J. A.; Callow, M. E.; Schultz, M. P.; Swain, G. W.; Callow, J. A. Adhesion Strength of Settled Spores of the Green Alga Enteromorpha. Biofouling 2002, 18, 251-256.

(50) K. Ista, L. Attachment of bacteria to model solid surfaces: Oligo(ethylene glycol) surfaces inhibit bacterial attachment. FEMS Microbiology Letters 1996, 142, 59-63.

(51) Schilp, S.; Kueller, A.; Rosenhahn, A.; Grunze, M.; Pettitt, M. E.; Callow, M. E.; Callow, J. A. Settlement and adhesion of algal cells to hexa(ethylene glycol)-containing self-assembled monolayers with systematically changed wetting properties. Biointerphases 2007, 2, 143-150. 
(52) Nolte, K. A.; Schwarze, J.; Rosenhahn, A. Microfluidic accumulation assay probes attachment of biofilm forming diatom cells. Biofouling 2017, 33, 531-543. 


\section{Table of contents}
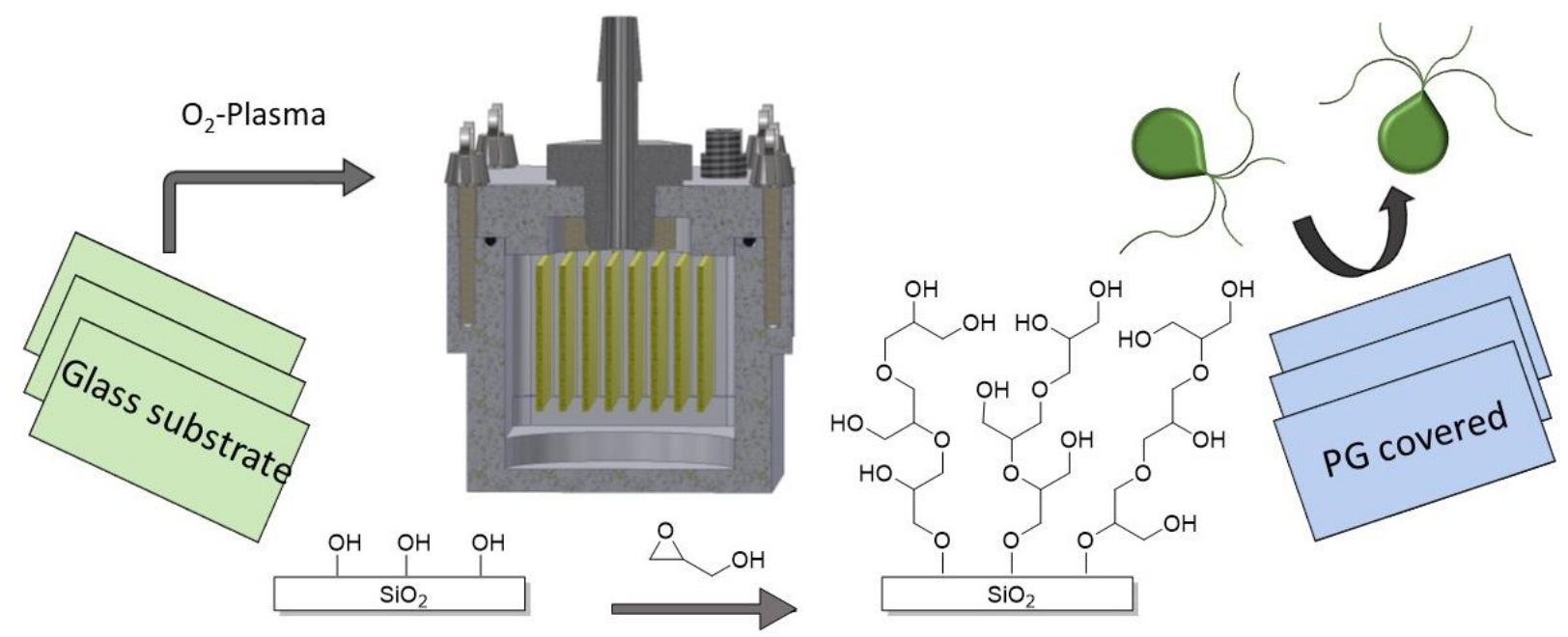

Dendritic polyglycerol coatings prepared via surface initiated ring-opening polymerization were discovered as marine fouling-release coatings.

High-quality dendritic polyglycerol coatings were applied to surfaces using a custom build Teflon reaction vessel. In both, laboratory diatom and in dynamic field accumulation assays, we found excellent fouling release behavior. The antifouling potential was supported by a high stability in sea water and a good protein-resistance, suggesting to consider these chemical components as ingredients for modern FR-coatings. 